\title{
Endocervical Gel Dosage Form
}

National Cancer Institute

\section{Source}

National Cancer Institute. Endocervical Gel Dosage Form. NCI Thesaurus. Code C149485.

Semi-solid preparation consisting of a gel intended for endocervical use by means of a suitable applicator. 